For quantitative comparison between the simulations we used statistical measures including correlation coefficient (R), root mean square error (RMSE), mean average error (MAE), mean bias $(\mathrm{MB})$, normalized mean bias (NMB), and index of agreement (IOA). Definitions of these metrics can be found below:

$$
\begin{aligned}
& R=\frac{\overline{\left(C_{o}-\overline{C_{o}}\right)\left(C_{p}-\overline{C_{p}}\right)}}{\sigma_{C p} \sigma_{C o}} \\
& R M S E=\sqrt{\frac{\sum_{i=1}^{n}\left(C_{p}-C_{o}\right)^{2}}{n}} \\
& M A E=\frac{1}{n} \sum_{i=1}^{n}\left|C_{p}-C_{o}\right| \\
& M B=\frac{1}{n} \sum_{i=1}^{n}\left(C_{p}-C_{o}\right) \\
& N M B=\frac{\left(\overline{C_{p}}-\overline{C_{o}}\right)}{\overline{C_{o}} \times 100 \%} \\
& I O A=1-\frac{\sum_{i=1}^{n}\left(C_{p}-C_{o}\right)^{2}}{\sum_{i=1}^{n}\left(\left|C_{p}-\overline{C_{o}}\right|+\left|C_{o}-\overline{C_{o}}\right|\right)^{2}}
\end{aligned}
$$

Where $C_{o}$ is the observation value, $C_{p}$ is the model value, $\sigma$ is the standard deviation, and $\bar{C}$ is the mean value 
Table SM 1. Summary of model performance in capturing temperature at BAO $10 \mathrm{~m}$ and $300 \mathrm{~m}$ during Aug 1-15, 2014

\begin{tabular}{|c|c|c|c|c|c|c|c|c|c|}
\hline \multirow[b]{2}{*}{$T(C)-10 m$} & \multicolumn{3}{|c|}{ PBL } & \multicolumn{2}{|c|}{ Met IC and BC } & \multicolumn{2}{|c|}{ Initialization } & \multicolumn{2}{|c|}{ Horizontal resolution } \\
\hline & PBL1 & PBL2 & PBL3 & Met5 & Met6 & Init4 & Init5 & Hor5 & Hor5-12km \\
\hline Mean Model & 22.40 & 20.95 & 21.20 & 24.06 & 23.44 & 21.59 & 24.06 & 24.06 & 24.08 \\
\hline Mean Obs & 21.67 & 21.67 & 21.67 & 21.67 & 21.67 & 21.67 & 21.67 & 21.67 & 21.67 \\
\hline $\mathbf{R}$ & 0.89 & 0.89 & 0.89 & 0.86 & 0.89 & 0.71 & 0.86 & 0.86 & 0.88 \\
\hline RMSE & 2.05 & 2.03 & 2.01 & 3.25 & 2.63 & 2.99 & 3.25 & 3.25 & 3.18 \\
\hline MAE & 1.56 & 1.62 & 1.59 & 2.60 & 2.05 & 2.30 & 2.60 & 2.60 & 2.53 \\
\hline MB & 0.74 & -0.72 & -0.46 & 2.40 & 1.77 & -0.08 & 2.40 & 2.40 & 2.41 \\
\hline NMB & $3.4 \%$ & $-3.3 \%$ & $-2.1 \%$ & $11.1 \%$ & $8.2 \%$ & $-0.4 \%$ & $11.1 \%$ & $11.1 \%$ & $11.1 \%$ \\
\hline IAO & 0.94 & 0.94 & 0.94 & 0.85 & 0.90 & 0.83 & 0.85 & 0.85 & 0.86 \\
\hline$T(C)-300 m$ & PBL1 & PBL2 & PBL3 & Met5 & Met6 & Init4 & Init5 & Hor5 & Hor5-12km \\
\hline Mean Model & 21.91 & 20.95 & 21.30 & 23.58 & 22.89 & 20.31 & 23.58 & 23.58 & 23.52 \\
\hline Mean Obs & 21.68 & 21.68 & 21.68 & 21.68 & 21.68 & 21.68 & 21.68 & 21.68 & 21.68 \\
\hline $\mathbf{R}$ & 0.76 & 0.75 & 0.72 & 0.74 & 0.78 & 0.57 & 0.74 & 0.74 & 0.75 \\
\hline RMSE & 2.16 & 2.14 & 2.10 & 2.79 & 2.27 & 3.09 & 2.79 & 2.79 & 2.80 \\
\hline MAE & 1.69 & 1.73 & 1.68 & 2.24 & 1.76 & 2.45 & 2.24 & 2.24 & 2.21 \\
\hline MB & 0.23 & -0.73 & -0.38 & 1.90 & 1.22 & -1.37 & 1.90 & 1.90 & 1.85 \\
\hline NMB & $1.1 \%$ & $-3.4 \%$ & $-1.8 \%$ & $8.8 \%$ & $5.6 \%$ & $-6.3 \%$ & $8.8 \%$ & $8.8 \%$ & $8.5 \%$ \\
\hline IAO & 0.87 & 0.85 & 0.84 & 0.78 & 0.85 & 0.72 & 0.78 & 0.78 & 0.79 \\
\hline
\end{tabular}


Table SM 2. Summary of model performance in capturing relative humidity (RH) at BAO $10 \mathrm{~m}$ and $300 \mathrm{~m}$ during Aug 1-15, 2014

\begin{tabular}{|c|c|c|c|c|c|c|c|c|c|}
\hline \multirow[b]{2}{*}{ RH (\%)-10m } & \multicolumn{3}{|c|}{ PBL } & \multicolumn{2}{|c|}{ Met IC and BC } & \multicolumn{2}{|c|}{ Initialization } & \multicolumn{2}{|c|}{ Horizontal resolution } \\
\hline & PBL1 & PBL2 & PBL3 & Met5 & Met6 & Init4 & Init5 & Hor5 & Hor5-12km \\
\hline Mean Model & 46.85 & 57.59 & 55.78 & 32.65 & 39.87 & 59.36 & 32.65 & 32.65 & 32.89 \\
\hline Mean Obs & 46.47 & 46.47 & 46.47 & 46.47 & 46.47 & 46.47 & 46.47 & 46.47 & 46.47 \\
\hline $\mathbf{R}$ & 0.78 & 0.69 & 0.73 & 0.63 & 0.64 & 0.53 & 0.63 & 0.63 & 0.71 \\
\hline RMSE & 10.89 & 16.90 & 15.13 & 19.13 & 14.95 & 22.33 & 19.13 & 19.13 & 18.15 \\
\hline MAE & 8.45 & 14.38 & 12.86 & 15.01 & 11.31 & 18.10 & 15.01 & 15.01 & 14.43 \\
\hline MB & 0.38 & 11.12 & 9.31 & -13.81 & -6.60 & 12.90 & -13.51 & -13.51 & -13.58 \\
\hline NMB & $0.8 \%$ & $23.9 \%$ & $20.0 \%$ & $-29.7 \%$ & $-14.2 \%$ & $27.7 \%$ & $-29.7 \%$ & $-29.7 \%$ & $-29.2 \%$ \\
\hline IAO & 0.88 & 0.74 & 0.78 & 0.65 & 0.75 & 0.65 & 0.65 & 0.65 & 0.69 \\
\hline RH (\%)-300m & PBL1 & PBL2 & PBL3 & Met5 & Met6 & Init4 & Init5 & Hor5 & Hor5-12km \\
\hline Mean Model & 43.63 & 51.45 & 48.25 & 31.27 & 38.55 & 59.06 & 31.27 & 31.27 & 31.94 \\
\hline Mean Obs & 38.70 & 38.70 & 38.70 & 38.70 & 38.70 & 38.70 & 38.70 & 38.70 & 38.70 \\
\hline $\mathbf{R}$ & 0.64 & 0.59 & 0.48 & 0.53 & 0.52 & 0.41 & 0.53 & 0.53 & 0.57 \\
\hline RMSE & 13.06 & 17.92 & 15.25 & 12.66 & 11.14 & 28.39 & 12.66 & 12.66 & 12.11 \\
\hline MAE & 9.92 & 14.78 & 12.77 & 9.73 & 8.60 & 23.19 & 9.73 & 9.73 & 9.29 \\
\hline MB & 4.93 & 12.75 & 9.55 & -7.43 & -0.15 & 20.36 & -7.43 & -7.43 & -6.76 \\
\hline NMB & $12.7 \%$ & $32.9 \%$ & $24.7 \%$ & $-19.2 \%$ & $-0.4 \%$ & $52.6 \%$ & $-19.2 \%$ & $-19.2 \%$ & $-17.5 \%$ \\
\hline IAO & 0.75 & 0.61 & 0.60 & 0.67 & 0.71 & 0.43 & 0.67 & 0.67 & 0.70 \\
\hline
\end{tabular}


Table SM 3 Summary of model performance in capturing wind speed and direction at BAO 10m during Aug 1-15, 2014

\begin{tabular}{|c|c|c|c|c|c|c|c|c|c|c|}
\hline & & \multicolumn{3}{|c|}{ PBL } & \multicolumn{2}{|c|}{ Met } & \multicolumn{2}{|c|}{ Init } & \multicolumn{2}{|c|}{ Horizontal Res. } \\
\hline \multicolumn{2}{|c|}{ Day - $10 m$} & PBL1 & PBL2 & PBL3 & Met5 & Met6 & Init4 & Init5 & Hor5 & Hor5-12km \\
\hline \multirow{4}{*}{ 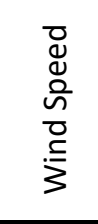 } & $\begin{array}{l}\text { Mean } \\
\text { Model }\end{array}$ & 2.99 & 2.68 & 2.20 & 2.63 & 2.83 & 3.30 & 2.63 & 2.63 & 2.58 \\
\hline & STD Model & 1.47 & 1.55 & 1.27 & 1.41 & 1.51 & 2.02 & 1.41 & 1.41 & 1.33 \\
\hline & Mean Obs & 2.46 & 2.46 & 2.46 & 2.46 & 2.46 & 2.46 & 2.46 & 2.46 & 2.46 \\
\hline & STD Obs & 1.25 & 1.25 & 1.25 & 1.25 & 1.25 & 1.25 & 1.25 & 1.25 & 1.25 \\
\hline \multirow{4}{*}{ لَّ } & $\begin{array}{l}\text { Mean } \\
\text { Model }\end{array}$ & 64.31 & 71.92 & 74.85 & 38.63 & 70.83 & 61.40 & 38.63 & 38.63 & 45.08 \\
\hline & STD Model & 45.40 & 62.30 & 54.02 & 73.77 & 75.30 & 75.65 & 73.77 & 73.77 & 66.18 \\
\hline & Mean Obs & 123.38 & 123.38 & 123.38 & 123.38 & 123.38 & 123.38 & 123.38 & 123.38 & 123.38 \\
\hline & STD Obs & 66.06 & 66.06 & 66.06 & 66.06 & 66.06 & 66.06 & 66.06 & 66.06 & 66.06 \\
\hline \multicolumn{2}{|c|}{ Night - $10 \mathrm{~m}$} & PBL1 & PBL2 & PBL3 & Met5 & Met6 & Init4 & Init5 & Hor5 & Hor5-12km \\
\hline \multirow{4}{*}{ 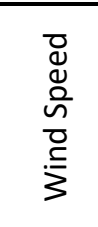 } & $\begin{array}{l}\text { Mean } \\
\text { Model }\end{array}$ & 2.81 & 2.58 & 2.18 & 2.51 & 2.72 & 2.91 & 2.51 & 2.51 & 2.66 \\
\hline & STD Model & 1.41 & 0.94 & 0.96 & 1.35 & 1.43 & 1.40 & 1.35 & 1.35 & 1.41 \\
\hline & Mean Obs & 2.25 & 2.25 & 2.25 & 2.25 & 2.25 & 2.25 & 2.25 & 2.25 & 2.25 \\
\hline & STD Obs & 0.96 & 0.96 & 0.96 & 0.96 & 0.96 & 0.96 & 0.96 & 0.96 & 0.96 \\
\hline \multirow{4}{*}{ لَّ } & $\begin{array}{l}\text { Mean } \\
\text { Model }\end{array}$ & 244.07 & 243.95 & 263.07 & 226.97 & 230.93 & 160.02 & 226.97 & 226.97 & 295.43 \\
\hline & STD Model & 90.68 & 69.52 & 74.66 & 83.89 & 69.81 & 87.15 & 83.89 & 83.89 & 87.30 \\
\hline & Mean Obs & 222.98 & 222.98 & 222.98 & 222.98 & 222.98 & 222.98 & 222.98 & 222.98 & 222.98 \\
\hline & STD Obs & 50.01 & 50.01 & 50.01 & 50.01 & 50.01 & 50.01 & 50.01 & 50.01 & 50.01 \\
\hline
\end{tabular}


Table SM 4 Summary of model performance in capturing wind speed and direction at BAO $300 \mathrm{~m}$ during Aug 1-15, 2014

\begin{tabular}{|c|c|c|c|c|c|c|c|c|c|c|}
\hline & & \multicolumn{3}{|c|}{ PBL } & \multicolumn{2}{|c|}{ Met } & \multicolumn{2}{|c|}{ init } & \multicolumn{2}{|c|}{ Horizontal Res. } \\
\hline \multicolumn{2}{|c|}{ Day - 300 m } & PBL1 & PBL2 & PBL3 & Met5 & Met6 & Init4 & Init5 & Hor5 & $\begin{array}{l}\text { Hor5- } \\
12 \mathrm{~km}\end{array}$ \\
\hline \multirow{4}{*}{ 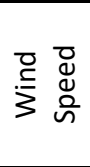 } & Mean Model & 3.89 & 3.51 & 2.78 & 2.88 & 3.22 & 3.83 & 2.88 & 2.88 & 2.77 \\
\hline & STD Model & 2.15 & 2.39 & 1.61 & 1.58 & 1.81 & 2.93 & 1.58 & 1.58 & 1.47 \\
\hline & Mean Obs & 3.23 & 3.23 & 3.23 & 3.23 & 3.23 & 3.23 & 3.23 & 3.23 & 3.23 \\
\hline & STD Obs & 2.24 & 2.24 & 2.24 & 2.24 & 2.24 & 2.24 & 2.24 & 2.24 & 2.24 \\
\hline \multirow{4}{*}{ 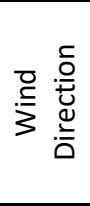 } & Mean Model & 62.69 & 62.42 & 64.05 & 32.91 & 57.67 & 56.71 & 32.91 & 32.91 & 39.52 \\
\hline & STD Model & 51.99 & 63.89 & 59.84 & 75.14 & 76.03 & 74.32 & 75.14 & 75.14 & 69.43 \\
\hline & Mean Obs & 117.31 & 117.31 & 117.31 & 117.31 & 117.31 & 117.31 & $\begin{array}{c}117.3 \\
1\end{array}$ & 117.31 & 117.31 \\
\hline & STD Obs & 74.56 & 74.56 & 74.56 & 74.56 & 74.56 & 74.56 & 74.56 & 74.56 & 74.56 \\
\hline \multicolumn{2}{|c|}{ Night - 300 m } & PBL1 & PBL2 & PBL3 & Met5 & Met6 & Init4 & Init5 & Hor5 & $\begin{array}{l}\text { Hor5- } \\
12 \mathrm{~km}\end{array}$ \\
\hline \multirow{4}{*}{ 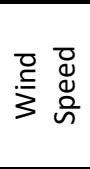 } & Mean Model & 5.00 & 4.34 & 3.80 & 4.21 & 4.60 & 5.07 & 4.21 & 4.21 & 4.89 \\
\hline & STD Model & 2.68 & 2.95 & 2.64 & 2.64 & 2.47 & 3.07 & 2.64 & 2.64 & 3.29 \\
\hline & Mean Obs & 3.42 & 3.42 & 3.42 & 3.42 & 3.42 & 3.42 & 3.42 & 3.42 & 3.42 \\
\hline & STD Obs & 2.59 & 2.59 & 2.59 & 2.59 & 2.59 & 2.59 & 2.59 & 2.59 & 2.59 \\
\hline \multirow{4}{*}{ 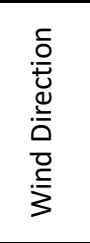 } & Mean Model & 141.12 & 223.36 & 355.95 & 326.05 & 294.02 & 156.88 & $\begin{array}{c}326.0 \\
5\end{array}$ & 326.05 & 306.58 \\
\hline & STD Model & 98.36 & 93.80 & 91.39 & 91.33 & 77.67 & 84.60 & 91.33 & 91.33 & 88.31 \\
\hline & Mean Obs & 213.59 & 213.59 & 213.59 & 213.59 & 213.59 & 213.59 & $\begin{array}{c}213.5 \\
9\end{array}$ & 213.59 & 213.59 \\
\hline & STD Obs & 72.73 & 72.73 & 72.73 & 72.73 & 72.73 & 72.73 & 72.73 & 72.73 & 72.73 \\
\hline
\end{tabular}



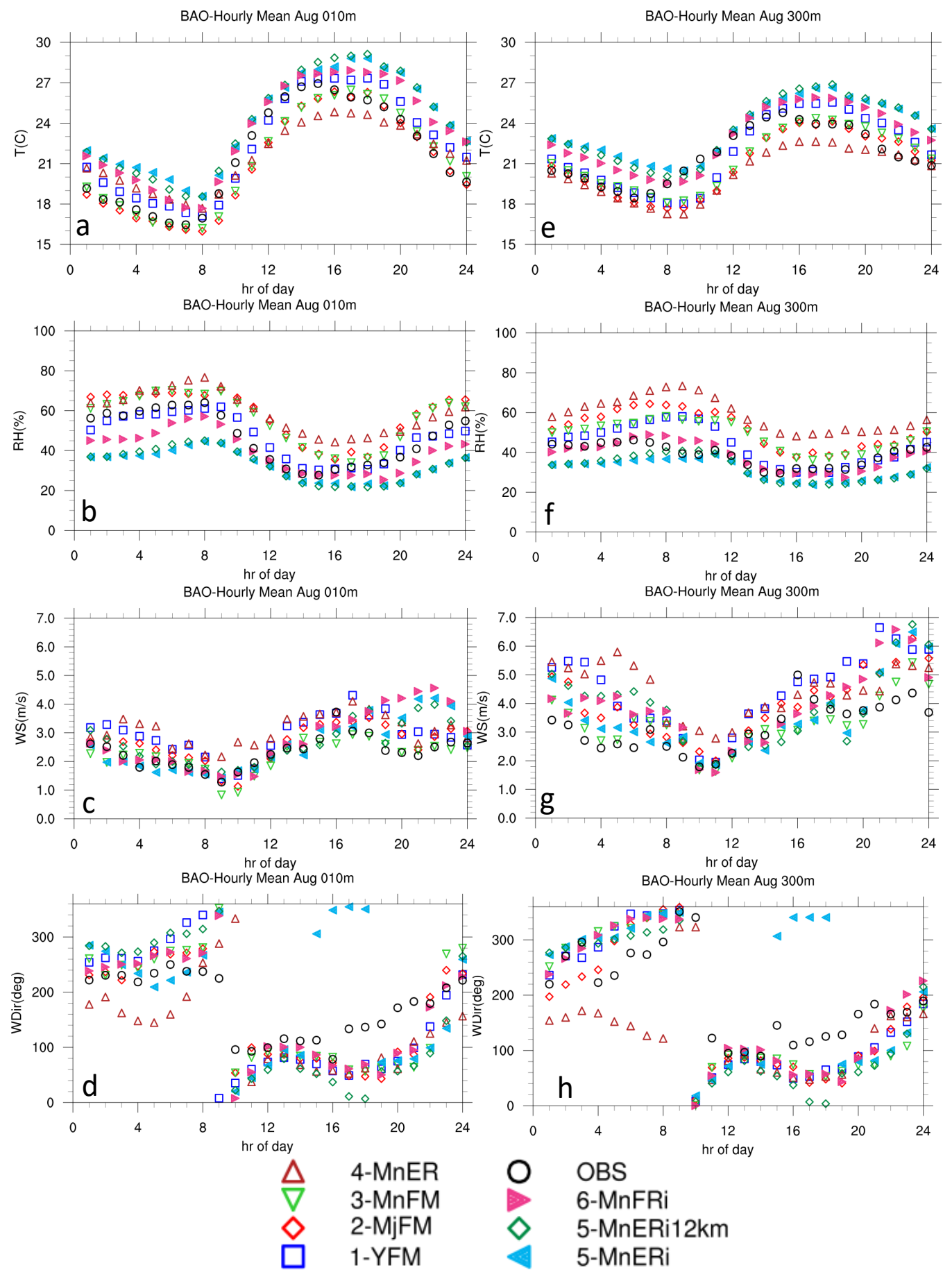

Figure SM 1. Average diurnal cycle of temperature $(a, e)$, relative humidity $(b, f)$, wind speed (c, $\mathrm{g}$ ) and wind direction $(\mathrm{d}, \mathrm{h})$ for all tests and observation at BAO $10 \mathrm{~m}$ and $300 \mathrm{~m}$. Averages are calculated for Aug 1 to 15, 2014. 

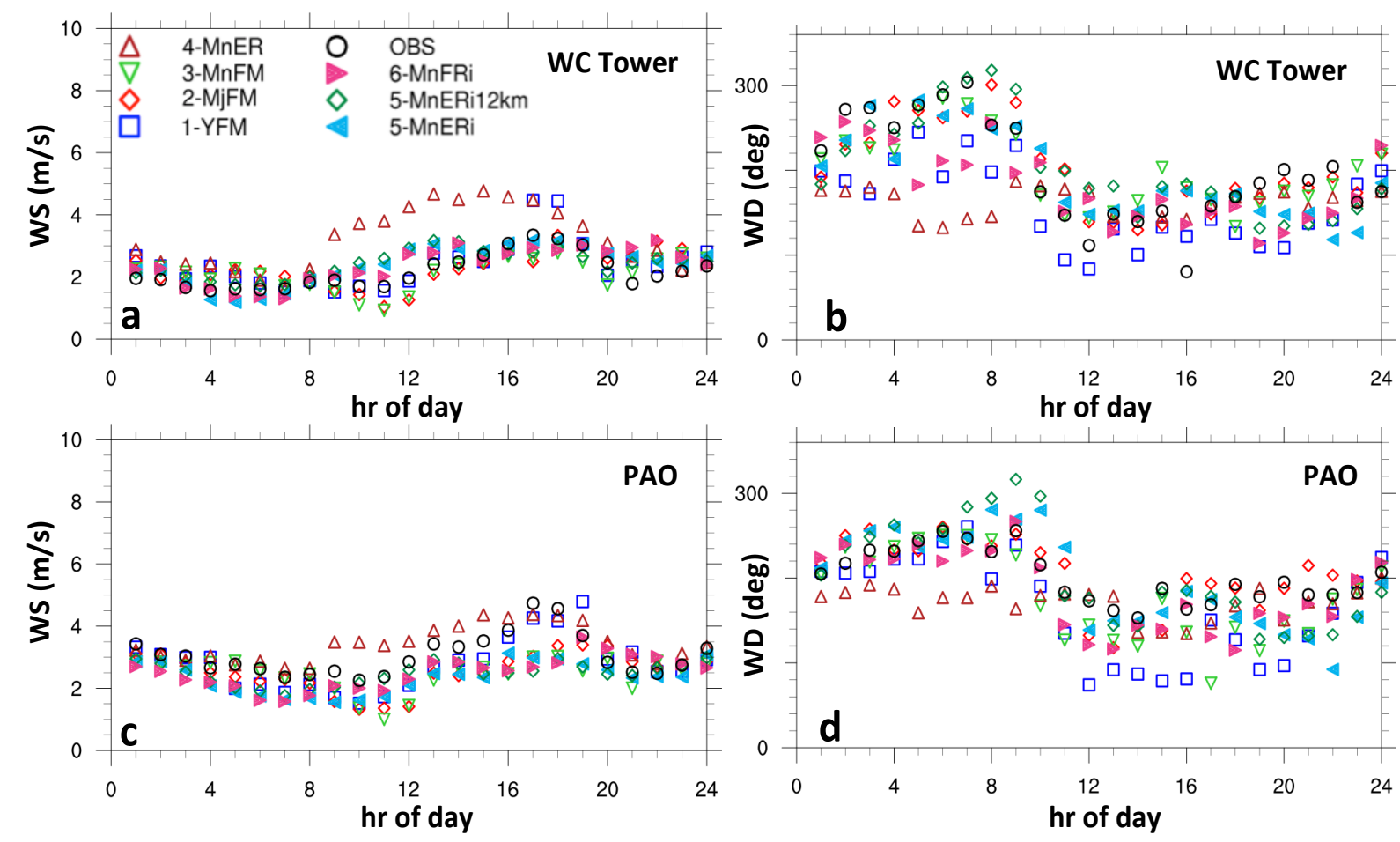

Figure SM 2. Average diurnal cycle of wind speed (WS) and direction (WD) at WC Tower and PAO sites. Averages are calculated for August 1 to 11, 2014. 


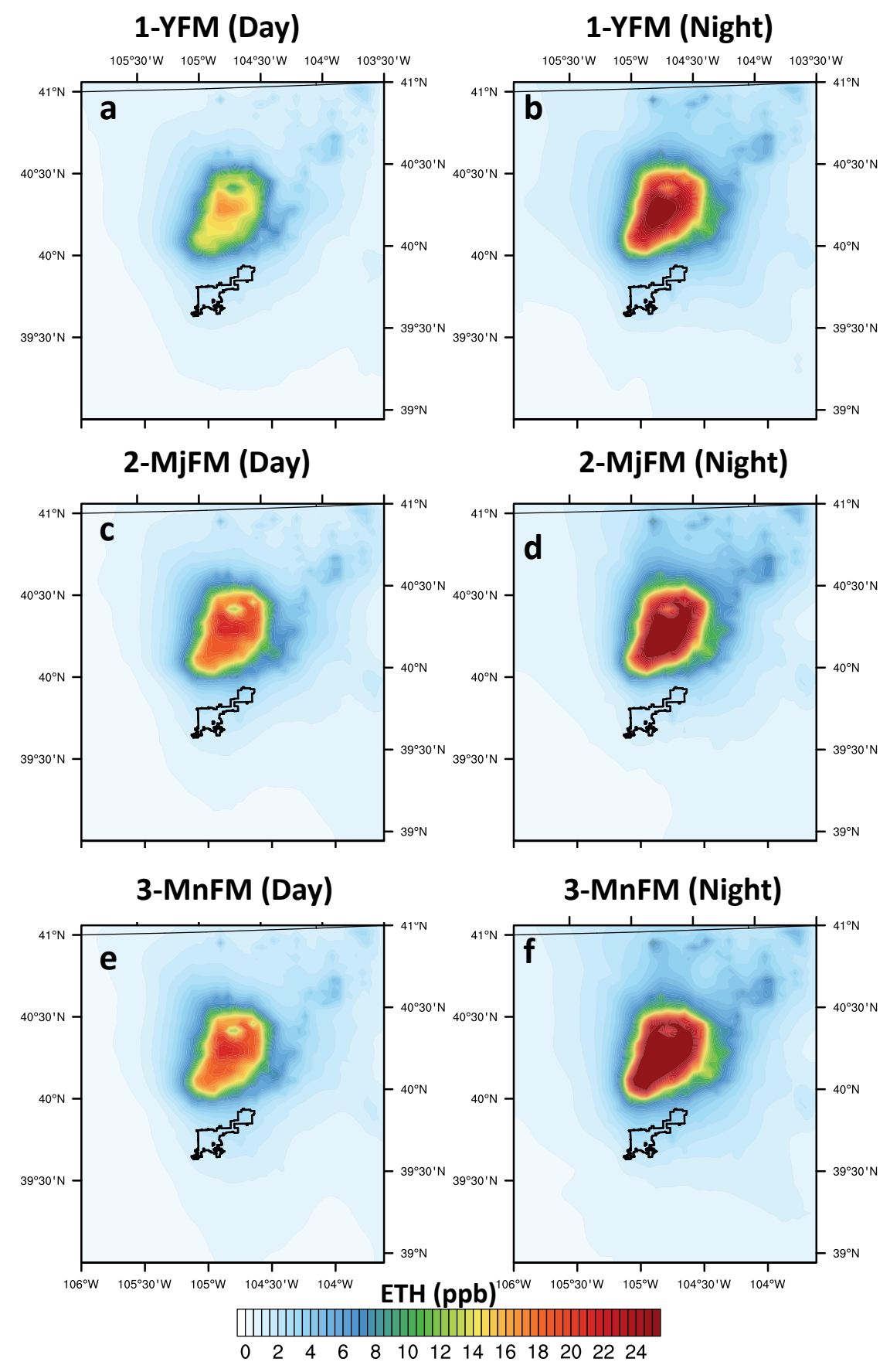

Figure SM 3. Surface ethane in sim 1 (1-YFM), sim 2 (2-MjFM), sim 3 (3-MnFm) averaged from August 1 to 15, 2014 


\section{4-07-28 (cyclone)}

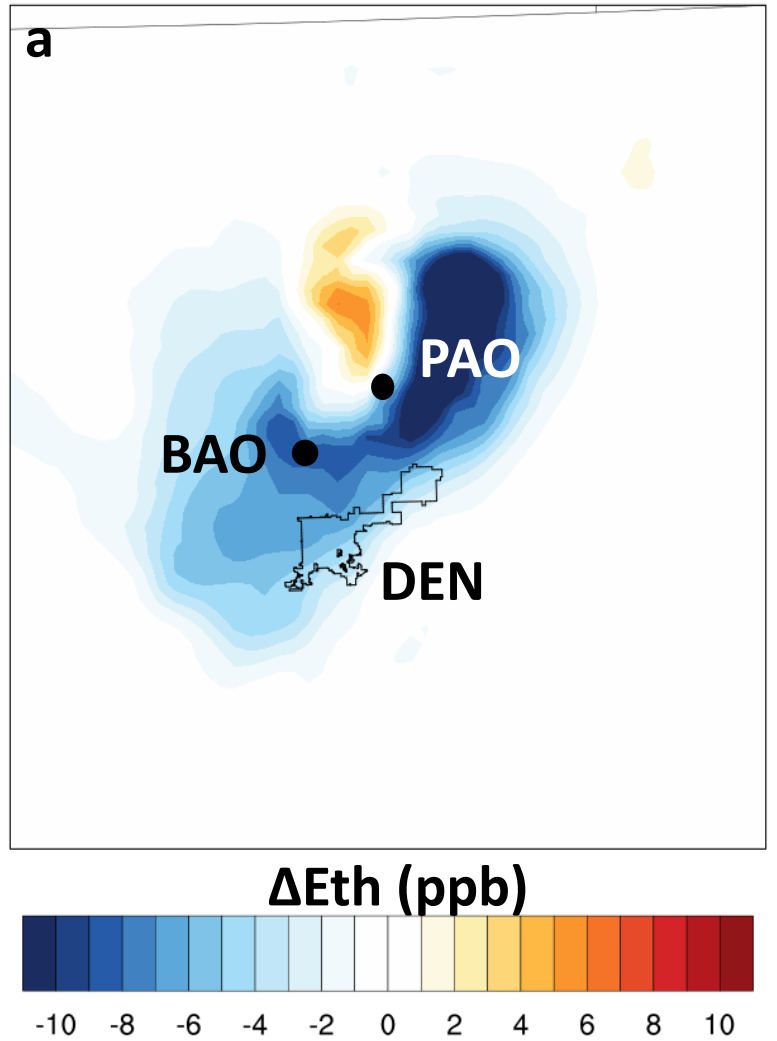

2014-08-02 (non-cyclone)

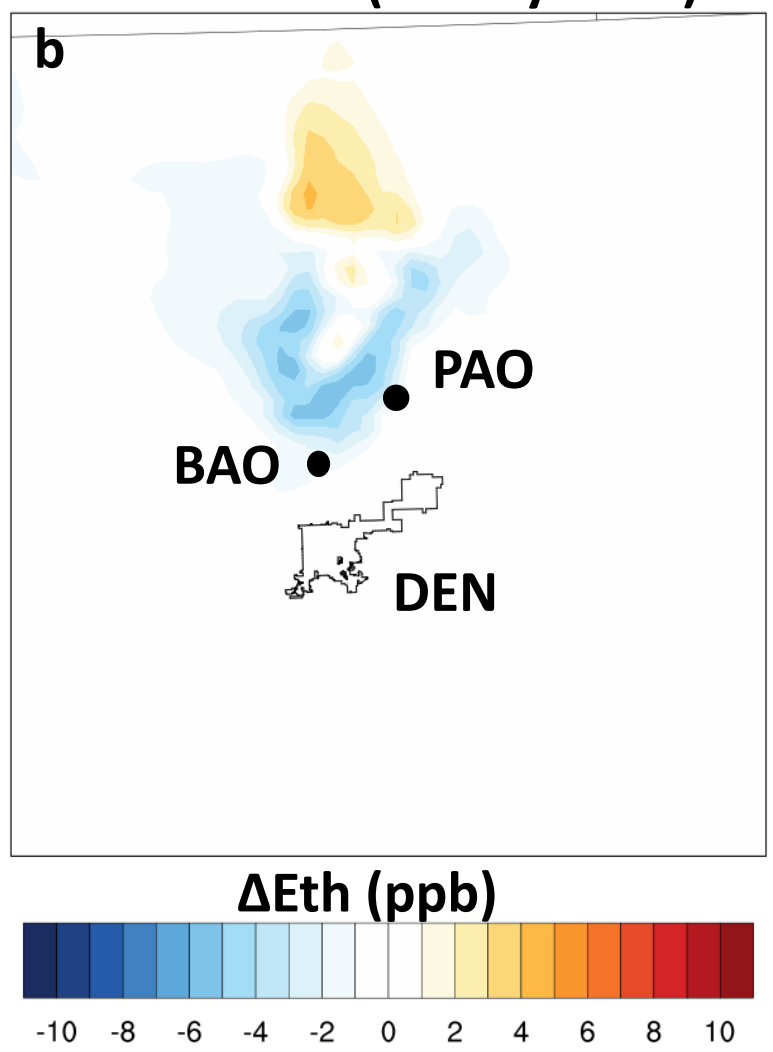

Figure SM 4. Difference in surface ethane concentration ( $\triangle \mathrm{Eth}$ ) between Met6 (6-MnFRi) and Met5 (5-MnERi) averaged during a) July 28, 2014 (Denver Cyclone) and b) August 2, 2014 (noncyclone). The locations of BAO and PAO sites and Denver county are marked on the map. 

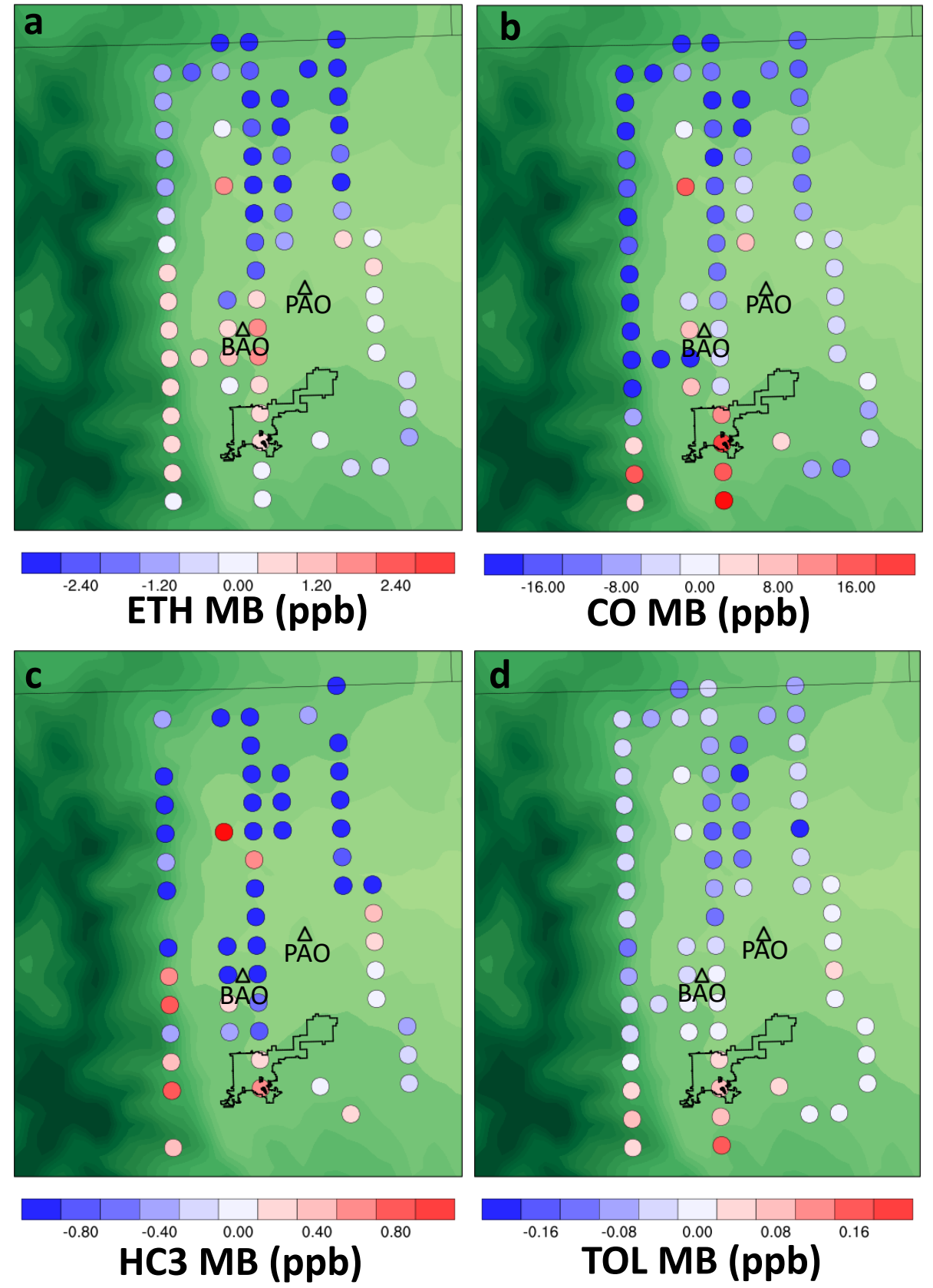

Figure SM 5. Mean biases of ethane (a), CO (b), HC3 (c), and TOL (d) in Em7 along the C130 PM flights limited to measurements below $1500 \mathrm{~m}$ agl and grids with more than 4 measurement points. Outline of the Denver county and location of BAO and PAO sites are marked on the underlying terrain map. 

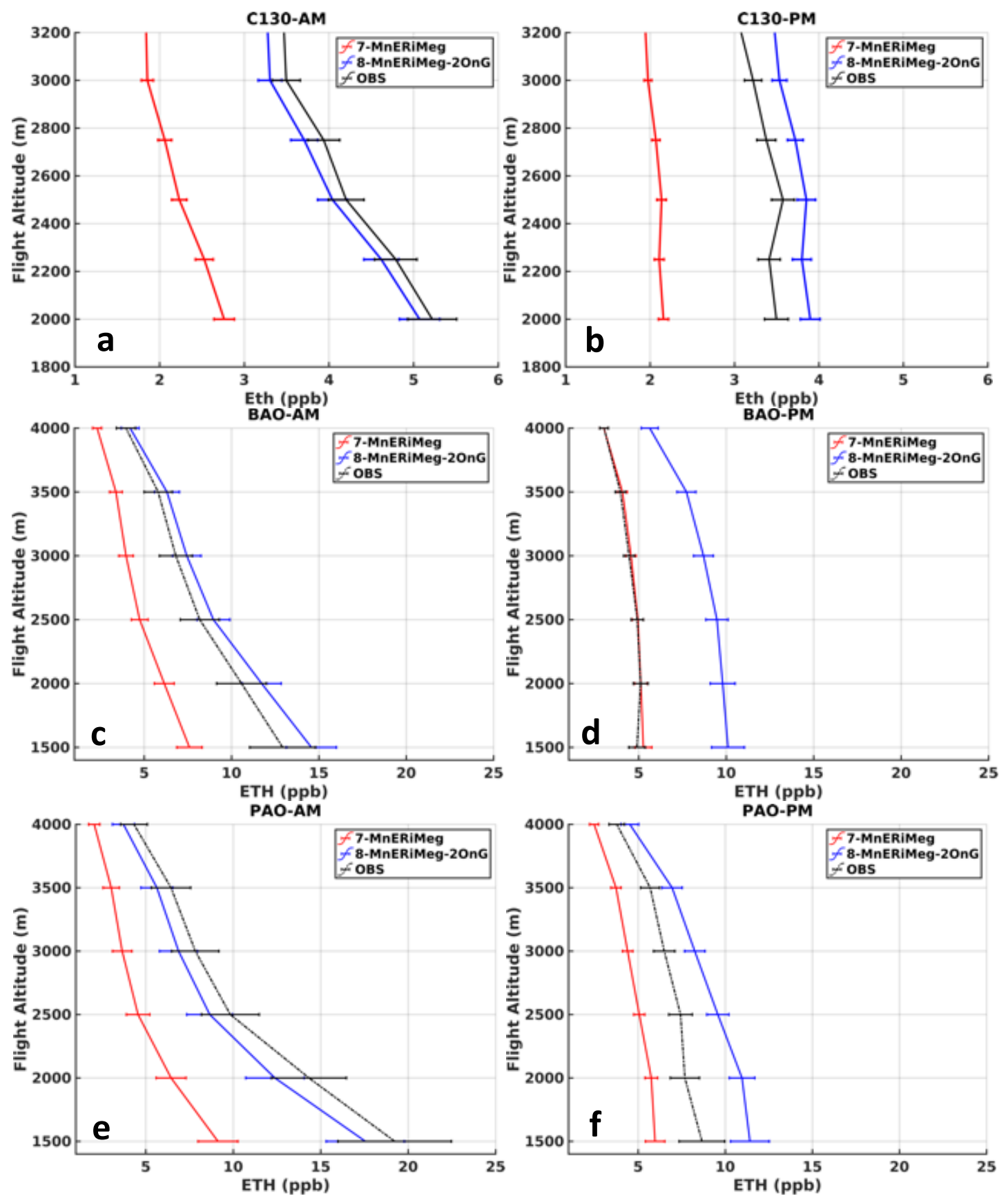

Figure SM 6. Sensitivity of ethane to oil and NG emission during C130-AM (a), C130-PM (b), P3PAO AM (d), P3-PAO PM (c), P3-BAO AM (e), P3-BAO PM (f) averaged for August flights. 